\title{
Evolutionary Control of Infectious Disease: Prospects for Vectorborne and Waterborne Pathogens
}

\author{
Paul W Ewald/ ${ }^{+}$, Jeremy B Sussman, Matthew T Distler, Camila Libel, Wahid \\ P Chammas, Victor J Dirita*, Carlos André Salles**, Ana Carolina Vicente***, \\ Ingrid Heitmann ${ }^{* * * *}$, Felipe Cabello*****
}

\begin{abstract}
Department of Biology, Amherst College, Amherst, MA 01002-5000, USA *Laboratory of Animal Medicine, University Michigan School of Medicine, Ann Arbor, MI 48109, USA **Departamento de Bioquímica e Biologia Molecular ***Departamento de Genética, Instituto Oswaldo Cruz, Av. Brasil 4365, 21045-900 Rio de Janeiro, RJ, Brasil ****Sub Departamento de Microbiologia Clinica, Instituto de Salud Publica, Maraton 1000, Nunoa Santiago, Chile *****Department of Immunology, New York Medical College, Vahalla, NY, USA

Evolutionary theory may contribute to practical solutions for control of disease by identifying interventions that may cause pathogens to evolve to reduced virulence. Theory predicts, for example, that pathogens transmitted by water or arthropod vectors should evolve to relatively high levels of virulence because such pathogens can gain the evolutionary benefits of relatively high levels of host exploitation while paying little price from host illness. The entrance of Vibrio cholerae into South America in 1991 has generated a natural experiment that allows testing of this idea by determining whether geographic and temporal variations in toxigenicity correspond to variation in the potential for waterborne transmission. Preliminary studies show such correspondences: toxigenicity is negatively associated with access to uncontaminated water in Brazil; and in Chile, where the potential for waterborne transmission is particularly low, toxigenicity of strains declined between 1991 and 1998. In theory vectorproofing of houses should be similarly associated with benignity of vectorborne pathogens, such as the agents of dengue, malaria, and Chagas' disease. These preliminary studies draw attention to the need for definitive prospective experiments to determine whether interventions such as provisioning of uncontaminated water and vector-proofing of houses cause evolutionary reductions in virulence.
\end{abstract}

Key words: infectious diseases - control - pathogens - waterborne transmission

\section{AN EVOLUTIONARY APPROACH TO VIRULENCE}

The ongoing synthesis of epidemiology, molecular biology, and evolutionary biology promises to improve our understanding of the temporal and geographic variation in pathogens and the diseases they cause. From a practical viewpoint this improved understanding may prove useful in identifying new possibilities for the control and prevention of infectious disease. One aspect of the infectious process that seems particularly amenable to this control is virulence, which is defined here as the level of harm to the host. Although viru-

This study was supported by two grants from Leonard $\mathrm{X}$ Bosack and Bette M Kruger Charitable Foundation (PWE), an Amherst College Faculty Research Award (PWE), a Hughes student fellowship (CL), and the Webster Fund of the Biology Department at Amherst College.

${ }^{+}$Corresponding author. Fax: +413-542.7955

Received 15 June 1998

Accepted 30 July 1998 lence depends on the interplay between pathogen and host characteristics, it is useful to consider the inherent virulence of a pathogen as the pathogen's contribution to this harmfulness. In practice this contribution is not separable from the host in which the harmfulness is assessed, yet conceptually reference to the inherent virulence of pathogens in the context of the spectrum of infectious agents. The smallpox viruses are inherently more harmful than rhinoviruses even though some of the mildest smallpox virus infections may be no more severe than the most severe rhinovirus infections.

Evolutionary considerations emphasize that the inherent virulence of pathogens should depend on a tradeoff between fitness benefits and fitness costs that are associated with particular levels of virulence. The fitness benefits are accrued through increased replication of the genetic instructions for the characteristic. Costs are typically accrued through reductions in the transmission of the genetic instructions, for example, due to negative effects of host illness on pathogen transmission. Evolutionary theory generally does not propose that virulence per se is beneficial. Rather, the logic 
assumes that disease organisms may benefit by exploiting their hosts. Such exploitation allows a disease organism to secure resources that it can use to reproduce, and thereby contribute more copies of the instructions for that exploitation into future generations. These fitness benefits of exploitation are weighed against the costs. The illness caused by intense levels of exploitation may make the host immobile, host mobility may be necessary for transmission to new hosts (as is the case, for example, with the common cold virus). In this case pathogen variants that exploit hosts so intensely that they cause host immobility may get more resources in the short run, but lose in the slightly longer run because of reductions in transmission. Pathogens that do not rely on host mobility for transmission pay a relatively low price if their exploitation immobilizes the host. According to the tradeoff reasoning presented above, pathogens in such categories should be particularly virulent. One of these categories involves waterborne transmission.

\section{WATERBORNE TRANSMISSION}

Waterborne transmission allows diarrheal pathogens to be transported from immobilized infected hosts to uninfected hosts. Where water supplies are not protected, a person with incapacitating diarrheal illness will release the diarrheal pathogens into clothes, bed sheets, or containers for collecting excreta. These items then tend to be removed by attendants and washed in bodies of water such as canals or rivers, which may be used as sources of drinking water or may flow into supplies of drinking water. Either way, the cycle is completed when susceptible individuals drink the contaminated water. In this situation, hightly exploitative (and hence highly virulent) pathogen variants should be favored by natural selection because the benefits of intense exploitation are great and the costs of exploitation are small. The benefits are great because large numbers of susceptibles can be infected by the increased numbers of propagules in the water. The costs are low because the incapacitating illness associated with this propagule production should have relatively little negative effect on the waterborne transmission of the propagules-rather than relying on the mobility of the infected individuals to enact transmission, the pathogens are using the mobility of the attendants and the water.

This hypothesized effect of waterborne transmission has been tested by determining whether the lethality of bacterial agents of human diarrhea is positively correlated with the degree to which they are waterborne (Ewald 1991). Fig. 1 shows that this correlation exists. Variation in the virulence of human diarrheal diseases can thus be explained in an evolutionary sense by variation in the degree to which different diarrheal pathogens are waterborne. This association offers some insight into the variation in virulence that occurs among diarrheal bacteria, but perhaps more importantly it suggests a new means for lessening the damage associated with diarrheal diseases. By reducing the potential for waterborne transmission we may be able to force diarrheal pathogens to evolve reduced virulence.

Whether this possibility is feasible depends on the validity of applying the trend apparent across the broad spectrum of diarrheal pathogens depicted in Fig. 1 to particular pathogens. Would a particular kind of pathogen evolve reduced virulence in response to a reduced potential for waterborne transmission? If so, what time period would be

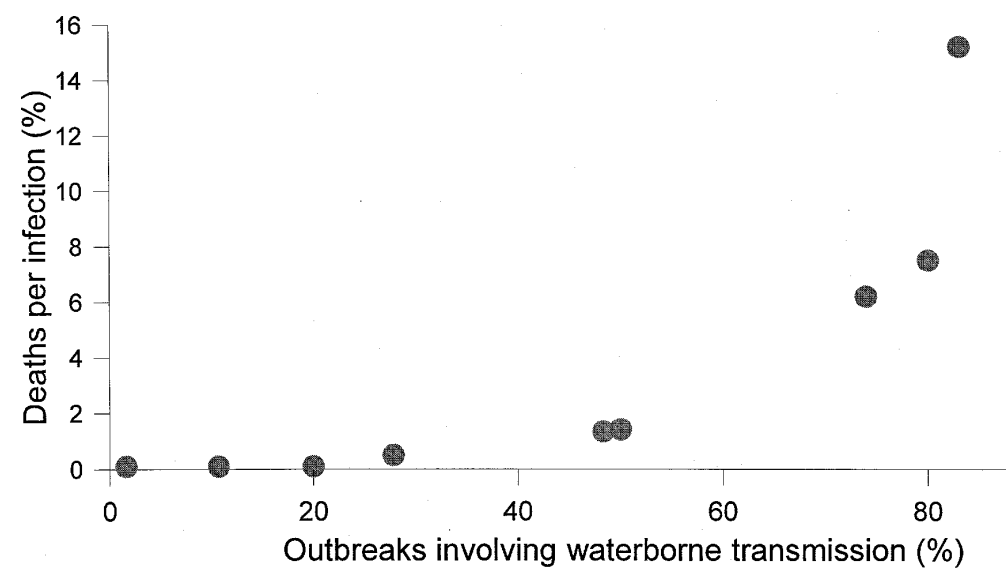

Fig. 1: waterborne transmission and mortality of diarrheal bacteria of humans. Pathogens ordered from most to least waterborne are classical Vibrio cholerae, Shigella dysenteriae type 1, Salmonella typhi, el tor V. cholerae, Shigella flexneri, Shigella sonnei, enterotoxigenic Escherichia coli, Campylobacter jejuni, and nontyphoid Salmonella (for other details see Ewald 1991). 
required? If this period were a few years, then efforts to improve access to clean water supplies would have evolutionary effects over an interval that is comparable to the intervals envisioned for conventional, nonevolutionary interventions.

$V$. cholerae is an excellent study subject for this kind of analysis particularly because its virulence is largely attributable to its toxin production. The inherent virulence of particular strains therefore can be assessed by quantifying levels of toxin production in vitro. Toxin production generates an efflux of fluid into the small intestine, which appears to provides two benefits to $V$. cholerae: (1) it flushes out competitors throughout the intestinal tract, allowing $V$. cholerae to pass down and out of the tract intact, and (2) it creates a fluid stool that probably facilitates transmission by contamination of the external environment and dissemination in water supplies. V. cholerae can persist in the intestine during this tumult because it can swim and adhere to the intestinal lining. The costs of toxin production include (1) the metabolic costs of producing the toxin and (2) the negative effect of toxin on host mobility and the probability of host death. Death from cholera results primarily from the dehydration which in turn results from the loss of fluid due to the toxin.

The cholera epidemic that has been unfolding in South America during the 1990s offers a natural experiment with which to assess the general theory. The first reported cases occurred in Peru at the beginning of 1991. The interval since then thus allows an assessment of whether any evolutionary effects of waterborne transmission can occur over a time interval comparable to the interval necessary for other categories of interventions such as vaccination or hygienic improvements to reduce the frequency of infection.

Within two years of the first reports of cholera from Peru the descendants of the Peruvian $V$. cholerae had spread from this epicenter throughout most countries of South and Central America (Tauxe et al. 1995). This spread set up a temporal and geographic pattern of infection that may allow detailed testing of the proposed evolutionary association between waterborne transmission and toxigenicity of $V$. cholerae.

We first focused on Brazil because water quality varies throughout Brazil, and the Brazilian Ministry of Health provides summaries of the proportion of the population with access to potable water. Moreover the large size of Brazil offers the potential for $V$. cholerae to evolve in different directions within the country. The first reported case of cholera in Brazil was in April 1991, about 2.5 months after the first reported case in Peru (Tauxe et al. 1995).
Although this analysis is still in progress, the results are consistent with an influence of water quality on virulence. If the mean for each state is used as a separate data point, there is a statistically significant negative association between access to potable water and $V$. cholerae toxigenicity (onetailed $\mathrm{p}<0.05$, Spearman $\mathrm{r}_{\mathrm{s}}=-0.62$ ). These data are, however, preliminary in several respects: (1) additional strains need to be obtained to make the accuracy of each data point more comparable. Some data points are based on multiple isolates others are based on only one isolate; (2) each strain was considered to be an independent data point in the statistical test; however, the degree to which the different data points are independent is unknown. Use of molecular phylogenies should allow the generation of tests that use independent pair-wise comparisons (Harvey \& Pagel 1991). This kind of comparison should be feasible eventually, but will probably need to be unusually extensive because nucleotide sequencing and pulsed field gel electrophoretic studies to date have detected almost no variation among the pandemic el tor strains (Salles \& Momen 1991, Karaolis et al. 1995); (3) changes in toxigenicity need to be tracked to determine whether harmful strains that enter areas with relatively pure water evolve reduced virulence over time.

Although the data from Brazil suggest that $V$. cholerae has evolved toward a lower level of virulence, they do not indicate how mild it could eventually become in response to cycling in areas with uncontaminated drinking water. To provide such an indication, Fig. 2 also plots the rate of toxin production of strains isolated from Texas and Louisiana in coastal areas of the Gulf of Mexico where $V$. cholerae has been endemic. Zymodeme analysis indicates that these US strains cluster with the el tor strains of $V$. cholerae (rather than strains of the classical biotype) but are only distantly related to these "mainstream" el tor strains (Salles \& Momen 1991). They therefore appear to have been present in the US for decades, perhaps being the remnant of a global outbreak of cholera that occurred many decades ago. Their low toxigenicity provides an indication of how low $V$. cholerae toxigenicity could become in an area with uncontaminated of drinking water. Accordingly, although the frequency of seropositivity to $V$. cholerae in local populations in this coastal area of the Gulf of Mexico can be substantial (MM Levine, personal communication), cases of cholera there are rare. Only about 50 cases have been reported from this region from 1965 through 1991 (Weber et al. 1994).

We are currently evaluating whether toxigenicity declines over time in regions with a low potential for waterborne transmission after $V$. cholerae 


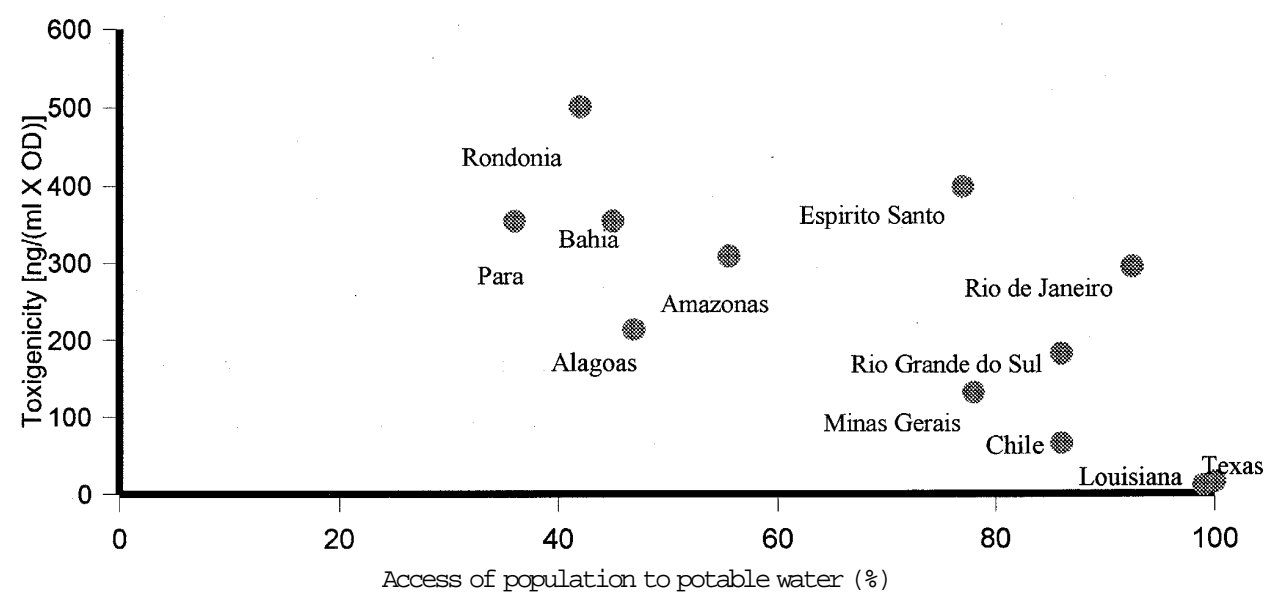

Fig. 2: toxigenicities of el tor Vibrio cholerae from Brazil, Chile, and the United States. Toxigenicities were assayed using standard ELISA techniques and AKI growth conditions. Names of states are given next to the data point that corresponds to the geometric mean toxigenicity of the strain(s) isolated from the state. Numbers next to each data point refer to the number of different strains tested. About 20 separate measurements of toxigenicity were made for each strain. The geometric mean toxin production was calculated for each strain. When more than one strain was obtained for a state, the geometric mean of the strain means was calculated. Although the V. cholerae strains from the United States are only distantly related to those circulating in Brazil, the US strains are presented to provide a sense of how benign strains of $V$. cholerae might become if they are exposed for a long period of time to clean water supplies. The Chilean strains are presented to illustrate how the mean generated from a collection of data associated with a reduction in toxigenicity through time corresponds with the overall geographic trend (see Fig. 2 and text).

enters such areas from regions with a higher potential for waterborne transmission. Our most complete data set in this regard, although still preliminary, comes from Chile. Chile is a particularly important country for evaluation of this hypothesis because it has one of the lowest potentials for waterborne transmission among Latin American countries for which el tor $V$. cholerae infections have become endemic. There is good access to uncontaminated drinking water and a steep elevational gradient that would limit cycles of waterborne transmission. V. cholerae entered Chile from Peru at the onset of the pandemic, the first case being reported from Chile about ten weeks after the first case was reported in Peru (Tauxe et al. 1995). Water supplies have been more contaminated in Peru than in Chile since the onset of the South American epidemic.

We have obtained and tested eight Chilean strains isolated from clinical cases over a time span that ranged from 1991 (the first year of the South American epidemic) to the beginning of 1998 . The toxigenicity of the tested Chilean strains dropped significantly as a function of time (Fig. 3, one-tailed $\mathrm{p}<0.02$, Spearman $\mathrm{r}_{\mathrm{s}}=-0.81$ ).

The geometric mean toxin production of the Chilean strains presented in Fig. 3 is also given in Fig. 2 to allow an assessment of the degree to which their toxigenicity conforms to that of the Brazilian and North American strains (for all data in Fig. 2;
Spearman, $\mathrm{p}<0.001, \mathrm{r}_{\mathrm{s}}=-0.75 ; \mathrm{N}=11$; the Louisiana and Texas values were treated as a single data point as a conservative measure).

The data from the most recent Chilean isolates are particularly interesting in light of the toxigenicities of the strains isolated in recent years, which are nearly as low as those isolated from the Gulf coast of the US (compare the values for the Chilean isolates of 1998 with those for the Texas and Louisiana). The drop in toxigenicity in Chile corresponds to a very low number of cholera cases. In 1994, for example, when nearly 50,000 cases were reported in Brazil and nearly 25,000 in Peru, the number of reported cases in Chile dropped to one (Tauxe et al. 1995). These figures coupled with the similar differences between the toxigenicities of the US Gulf strains and the most recent Chilean strains further supports the idea that the evolutionary management of virulence is feasible for $V$. cholerae if the potential for waterborne transmission can be sufficiently reduced.

At least four explanations exist for the evolution of reduced toxigenicity of $V$. cholerae in response to reduction in waterborne transmission: (1) the reduction in toxigenicity could result from the increased costs and decreased benefits of toxin production as outlined above; (2) the reduction could result from a variation on this theme, in which the growth of $V$. cholerae in marine environments disfavors toxigenicity, much in the same way the 
culturing of parasites outside of hosts causes evolutionary attenuation when genes for virulence no longer provide a fitness benefit to the organism; (3) the decline in Chile could be interpreted as a result of the duration of time that the outbreak had been cycling. Theory suggests that as an outbreak becomes endemic, pathogens might evolve reduced virulence (Lenski \& May 1994). To evaluate this hypothesis analogous data are needed from "control" countries invaded by V. cholerae at the same time, but for which water quality has remained low. If the reduction in toxigenicity of $V$. cholerae in Chile is attributable at least in part to its low potential for waterborne transmission, this reduction should be stronger than that found in such control countries. We have not yet obtained such a data set, but this comparison is feasible because strains of $V$. cholerae have been isolated in various countries throughout the pandemic; (4) the decrease in waterborne transmission might favor decreased virulence by reducing the genetic heterogeneity of the population of pathogens within a host. Although this hypothesis is probably generally applicable across a broad range of disease organisms, it does not appear to be particularly applicable to $V$. cholerae because its pathogenicity does not involve direct use of host resources, but instead involves the secretion of a product that benefits all of the other V. cholerae in the intestinal lumen (see the description of toxin action presented above).

Additional studies are needed to assess these four alternatives. It should be noted however, that from the practical perspective of evolutionary control of disease virulence, the precise mechanism is not so critical as recognition of the association.
That is, whatever combination of these explanations is correct, virulence of $V$. cholerae would still evolve toward lower levels in esponse to investments that reduce waterborne transmission.

The comments about phylogenetically paired comparisions mentioned in the context of Fig. 2 also apply to the data in Fig. 3, and neither data set controls for several other variables. Being based on strains that have been isolated and archived, the comparisons do not control, for example, for the source of material. The source of all or virtually all of the strains was clinical material, but the source was often not recorded explicitly in the archived information. Nor was the gathering of strains regimented so as to eliminate gathering biases. Strains isolated at the onset of an outbreak might be disproportionately gathered from severe infections, because severe infections would attract the attention of investigators, who would then develop approaches during the outbreak that would generate samples that were more representative of the existing sample. Although this kind of sampling bias might have contributed to the trend presented in Fig. 3, particularly with regard to the high value for the 1991 isolate, a sampling bias seems inadequate as an explanation of the overall trend, which results from the extremely low levels of toxin production of the strains isolated during the last few years. The toxin production of these strains is one to two orders of magnitude below that of typical el tor strains. Any biases associated with identification of the early cases in the Chilean epidemic should not have created the uniformly low levels of toxin production that were associated with the strains during the latter half of the epidemic; more-

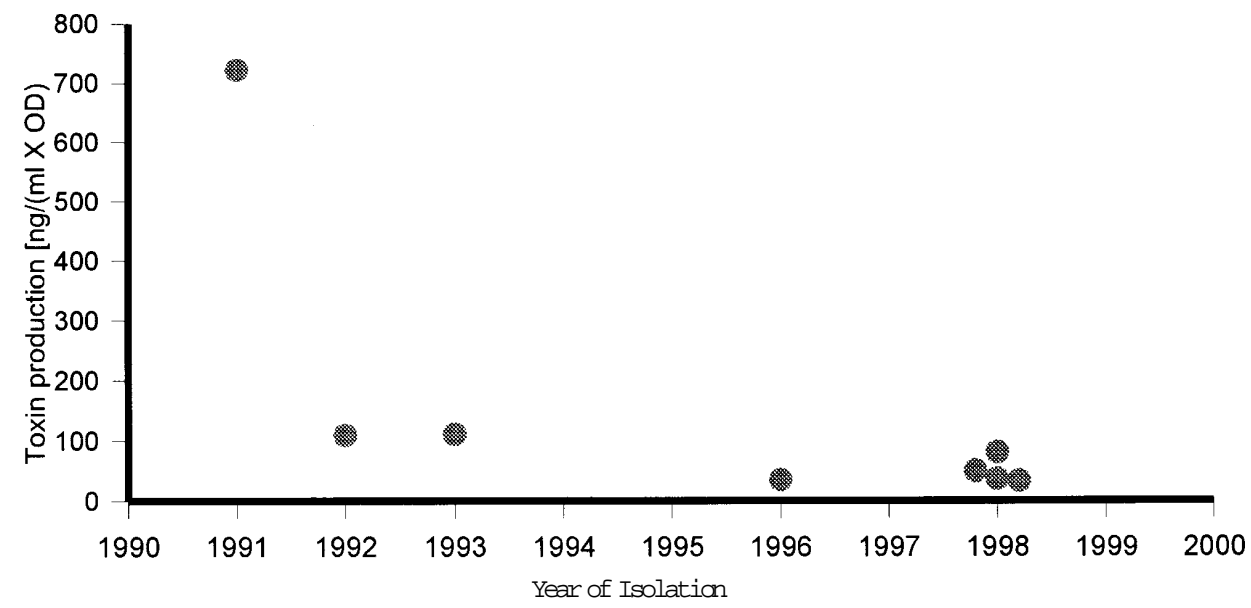

Fig. 3: toxigenicities of el tor Vibrio cholerae isolated from Chile from the beginning of the South American outbreak through the beginning of 1998. Each data point corresponds to a different isolate. Figures for access to water supplies are from ministries of health statistics for 1996 (Water access percentages from Louisiana and Texas are artificially separated to allow visualization of data points.). Other details are as described in Fig. 1. 
over, the statistical test used is sensitive relative rather than the absolute amounts of toxin production. If the 1991 strain from Chile had, for example, been only one-fifth of its measured value (and substantially less than a "typical" el tor strain), the statistical significance would have remained unchanged.

\section{VECTORBORNE TRANSMISSION}

Evolutionary theory identifies vectorborne transmission as a second factor favoring evolution toward relatively high levels of virulence. If a disease organism is transmitted by a biting arthropod vector such as a mosquito or reduviid bug, then it can still be transmitted even if a person is entirely immobilized with illness because such biting arthropods come to feed at immobile people. In fact, experimental studies indicate that mosquitoes are better able to bite a laboratory animal when it is sick with a vectorborne disease such as malaria than when it is healthy, and reduviid bugs (which are vectors for Chagas' disease) typically feed on sleeping individuals. As a consequence, natural selection should favor relatively high levels of host exploitation by vectorborne pathogens, and we should therefore see a particularly high virulence among vectorborne diseases.

The mortality associated with untreated infections is highly variable among both vectorborne and directly transmitted pathogens, but it is greater for vectorborne pathogens than for directly transmitted pathogens (Ewald 1983, 1994). Just as reduction of waterborne transmission should favor evolutionary decreases in virulence, reduction in the potential for arthropodborne transmission from immobilized humans should favor decreases in virulence. This effect can occur through two mechanisms. One mechanism is the direct analog of the argument for waterborne transmission, namely that reduction of transmission from immobilized humans causes a greater reliance on human mobility for transmission. Much as provisioning of uncontaminated drinking water is an intervention that should cause evolutionary reductions in the virulence of diarrheal pathogens, mosquito-proofing of houses is an intervention that should cause an evolutionary reduction in the virulence of vectorborne pathogens such as the agents of malaria or dengue. If a person ill with malaria or dengue stays in bed in a vector-proof house (or hospital), then the transmission of any pathogens in that person will be blocked during that period. To the extent that those variants tend to be inherently more virulent than variants that allow infectious people to be feeling well enough to move around outside of their homes, the composition of the pathogen population will shift toward a greater representation of the milder variants. That is, the pathogen population will have evolved toward mildness. This prediction has not yet been tested, but the information available in the literature, both supports the key steps in logic and suggests that the next stage of large-scale testing is warranted and would be beneficial even if the hypothesis is incorrect.

First, illness tend to be associated with infectiousness. For vectorborne viral diseases, such as dengue, the evidence is straight-forward: viremia occurs during the symptomatic period (e.g., Vaughn et al. 1977). In parasites with more complicated life histories, such as plasmodia, the evidence is more complex because the critical variable is the timing of infectious life history stages (i.e., the gametocytes) is the critical variable. In this case the evidence still supports the idea that much of the transmissibility will be associated with the period of reduced host mobility (e.g., see Ewald 1994).

Geographic variation indicates that parasites have the potential to cause largely mild infections where opportunities for vectorborne transmission are limited. $P$. vivax strains, for example, tend to be more mild in geographic areas associated with low and sporadic mosquito transmission (Ewald 1994). The variation in $P$. vivax's distribution appears to be largely a result of differences in the parasite's tendency to generate dormant resting stages (i.e., "hypnozoites").

$P$. falciparum infections are often similarly mild where the potential for vectorborne transmission is low, for example, in low transmission areas in the Sudan and Columbia (Elhassan et al 1995, Gonzalez et al. 1997). This tendency also occurs in Mali and more generally along the northern edge of $P$. falciparum's range in subsaharan Africa (D. S. Peterson, pers. comm.), where the parasite's distribution may be limited by the restricted abundance of mosquitoes. The relative importance of host and parasite characteristics in determining the mildness of $P$. falciparum infections has not been determined in any of these areas, however. If the mildness of such P. falciparum infections results at least in part from the mildness of the P. falciparum variants, evolution toward reduced virulence would seem particularly feasible. With pre-existing mild strains, detectable evolutionary shifts toward mildness could occur relatively quickly if mosquitoproofing programs were enacted at the edges of $P$. falciparum's distribution. If these programs proved successful the interventions could progress toward the center of the ranges, because the mild strains that would be needed to replace the more severe strains would already be present in the $P$. falciparum gene pool. Although such a progression might facilitate a rapid evolutionary shift to- 
ward benignity, it may not be necessary, as variations in pathogen virulence appear to be present even in areas with intense transmission (e.g., Kun et al. 1988).

Influences of exposure to infection on host resistance is a potential confounding variable in any efforts to control malaria through reduction in frequencies of transmission. One hypothesis attracting recent attention proposes that reductions in entomological inoculation rates (EIRs) will have little effect on overall mortality and morbidity in areas with moderate to high bite frequencies, where the benefits of reduced EIRs might be offset by reductions in acquired resistance (Snow \& Marsh 1995). With regard to evolutionary effects, this concern is applicable primarily to areas with moderate EIR. In areas with low EIR, mosquito-proofing should lower frequencies of infections to the point of eradication (Watson 1949). In areas with high EIRs, one would expect that mosquito-proofing would cause an evolutionary shift toward benignity with relatively little effect on frequency of infection, and hence with little effect on benefits of acquired immunity. If the evolutionary hypothesis is incorrect, great epidemiological benefits can be expected at least in areas with low EIRs; such nonevolutionary benefits at higher EIRs are uncertain. If the evolutionary hypothesis is correct this benefit at low EIRs will be supplemented with reduced virulence of infections across the spectrum of EIRs.

As is the case with waterborne transmission, vectorproofing of houses can be expected to provide evolutionary reductions in virulence across a spectrum of vectorborne diseases. Different strains of dengue, for example, vary in virulence, with the more virulent strains being more productive in cell culture (Morens et al. 1991). Vector-proofing of houses against dengue's vector, Aedes aegypti, should similarly favor the milder less exploitative variants, driving the dengue population to a more benign state. When more than one vectorborne disease is occurring in an area, the overall cost effectiveness may increase in proportion to the number of diseases, because the same intervention should have similar evolutionary effects for each.

The next stage of testing of these ideas will be feasible only if those who control the sources of funds consider the effort worthwhile. The chances of such a positive assessment would be improved if vector-proofing of houses could be shown to have traditional nonevolutionary epidemiological benefits (i.e., reduction in the frequency of infection) in addition to the hypothesized evolutionary epidemiological benefits (i.e., reduction in the harmfulness of the causative organisms). The available evidence indicates that traditional ben- efits do occur. The effectiveness of mosquito-proof housing against transmission of dengue, for example, is suggested by the resistance to invasion when such housing is generally present. Over the past two decades thousands of cases of dengue fever have occurred on the Mexican side of the US/ Mexico border along the Gulf of Mexico. Dengue has been introduced repeatedly into Texas there but has failed to spread in spite of the ubiquitous presence of Aedes vectors. For every reported case acquired on the Texas side of the border there are about 1000 reported cases on the Mexican side (CDC 1996). The pervasiveness of mosquito-proof on the Texas side appears to be responsible for this difference. Similarly, malaria has been introduced on numerous occasions in recent years to areas in the US where it had previously been endemic. Appropriate vectors are abundant, yet little secondary transmission occurs; when it does, it has been self-limited and localized (Wyler 1993, Dawson et al. 1997; for an analogous example involving severe diarrheal disease, see Weissman 1974 ).

The most thorough experimental test of the effectiveness of mosquito-proof housing on malaria transmission was conducted from 1939 through the 1940s in a large section of northern Alabama, by the Tennessee Valley Authority (TVA), which was overseeing the construction dams in the area (Watson 1949). The TVA was concerned about malaria because the construction of dams in the region had previously contributed to the malaria problem there (Ackerman 1956, Derryberry 1956).

During the 1930s about half of the people in the area tested positive. In 1939, the TVA began a campaign to mosquito-proof all houses in the area and accomplished this goal within seven years. They divided the area into 11 zones and completed the mosquito-proofing of each zone at different times. The results of their study show that mosquito-proofing virtually eradicated malaria from the area, with the decline occurring earlier in those zones in which mosquito-proofing was completed earlier (Fig. 4). No other intervention was enacted prior to the decline (Watson 1949).

These results do not represent a test of the idea that malaria pathogens evolve to lower levels of virulence in response to mosquito-proofing of houses. The results do, however, demonstrate several important points.

First, the results show that Plasmodium populations are influenced by mosquito-proofing. If the population as a whole declines so strongly in response to screening, it seems probable that certain variants within the population will be more substantially reduced by screening than others, leading to an evolutionary change in the Plasmodium gene pool. 


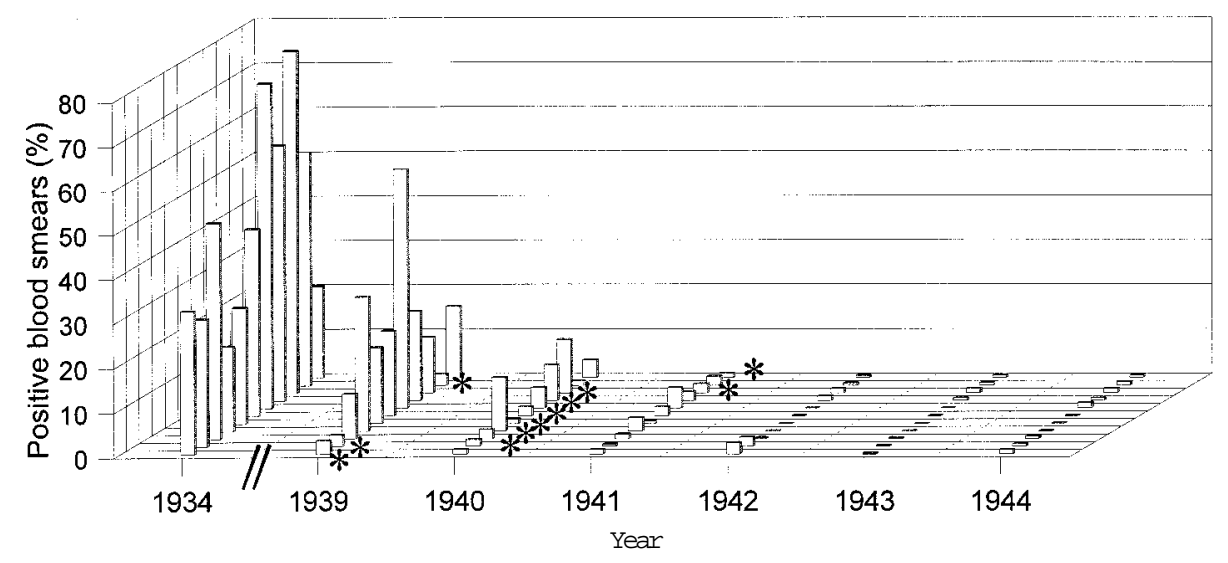

Fig. 4: seropositivity of blood samples for Plasmodium presented as a function of year during the mosquito-proofing program carried out in Alabama by the Tennessee Valley Authority. Each row corresponds to one of the 11 geographic zones that comprised the study. The asterisk designates the year in which mosquito-proofing was completed for all houses in the zone. See text for other details (data from Watson 1949).

Second, the results demonstrate nonevolutionary benefits necessary to justify the large-scale evolutionary experiment that would be needed to assess virulence management through mosquitoproofing. To justify the experiments from both ethical and economic perspectives, new areas for experimentation could be selected on the basis of having a slightly more difficult control problem than those for which nonevolutionary success has been demonstrated (e.g., a slightly higher prevalence of infection than occurred in northern Alabama just prior to the mosquito-proofing).

Third, the results show that the experiment is feasible logistically and financially even with the limitations of 1940s technology. The costs of mosquito-proofing (in 1944 dollars) was about $\$ 100$ per house for the area with the poorest quality of housing; the costs of maintaining the mosquitoproofing was about $\$ 12$ per house per year (Watson 1949). Modern technology has generated materials that are more effective, more durable, easier to apply and maintain, and more pleasant to live with than those used in the TVA study. Costs should therefore not be as greatly increased as would be indicated by a simple adjustment of the TVA costs for inflation. The actual costs may be influenced up or down depending on the details of a particular area such as the quality of existing houses, the degree to which materials could be generated locally and the costs of local labor.

Finally, the results of the TVA study demonstrate that mosquito-proofing worked even though this geographic area can be stiflingly hot and humid during the malaria season. Skeptics could have argued that people would not stay inside of houses sufficiently under such conditions for the antimalarial effects of mosquito-proof housing to work. Or, skeptics could have argued that people would deliberately destroy screens to increase air-flow through houses, but such vandalism was rare in the Alabama study (Watson 1949).

These ideas should be generally applicable across the spectrum of vectorborne diseases, although the particular details of the application will depend on the details of the vectorborne disease. Chagas disease offers an informative illustration of one variation on the theme. The agent of Chagas disease, Trypanosoma cruzi, is transmitted by reduviid bugs that bite sleeping individuals. It is therefore transmitted largely while people are immobilized in their houses. The frequencies of infection should therefore be reduced by vector-proofing of houses.

The details of $T$. cruzi transmission indicate that this intervention could reduce the virulence of $T$. cruzi through two evolutionary processes. The first process is analogous to that proposed above for malaria and dengue. To the extent that transmission does sometimes occur from mobile hosts outside of houses, virulence could be reduced.

The second evolutionary process concerns the effects of alternative vertebrate hosts on virulence in humans. The extent of human-bug-human transmission varies substantially geographically; substantial human-bug-human transmission occurring throughout most of $T$. cruzi's range but is virtually if not entirely absent in the US. Theory and comparative data indicate that vectorborne pathogens should tend to be relatively mild in humans when they rarely cycle in humans (Ewald 1983). About 
$5-25 \%$ of nonhuman vertebrate hosts (racoons and oppossums) in the southern US are infected with T. cruzi (Burkholder et al. 1980, Karsten et al. 1992, Pung et al. 1995), and a comparison of such strains with strains from humans in Brazil that they are genetically distinct (Clark \& Pung 1994). In the US humans rarely acquire $T$. cruzi via vectors, and appear to be dead-end hosts, probably because of the vector proof housing and low vector densities (Burkholder et al. 1980, Kirchhoff 1993, Barrett et al. 1997). In accordance with theory about the evolution of virulence, such infections appear to be particularly mild in humans, so much so that only three cases of acute Chagas' disease from bug bites had been reported in the US as of 1993 (Woody et al. 1961a, b, Kirchhoff 1993).

This situation is of importance to evolutionary control of $T$. cruzi in countries with endemic Chagas disease because by making houses vector-proof, the importance of human-bug-human cycling relative to enzootic cycling should become greatly reduced, thus causing the evolution of increased specialization of $T$. cruzi on nonhuman vertebrates, reduced specialization on humans, and consequently, reduced virulence in humans. As in the case of malaria, the presence of benign strains could be beneficial through protection against severe strains like a free live vaccine, because benign strains of $T$. cruzi can protect against highly virulent clones (Lauria Pires \& Teixeira 1997).

\section{THE MERGING OF EPIDEMIOLOGY WITH EVOLUTIONARY BIOLOGY}

The ideas presented above illustrate how the evolutionary considerations of virulence is bringing the health sciences is adding a new dimension to the ideas of the early epidemiologists. After Evandro Chagas deciphered the mode of transmission of $T$. cruzi, he stressed the importance of breaking the domestic cycle of transmission through the vector-proofing of houses. Decades later, in the 1940s, the architects of the mosquitoproofing campaign in Alabama stressed the same point for the control of malaria and demonstrated its utility (Watson 1949). But just as the results of the Alabama study were becoming available, DDT was introduced and successfully used to control malaria in the Mediterranean and South Asia (Harrison 1978). Also at that time the powerful quinine derivatives against malaria were being discovered in response to the cut-off of natural quinine to the Allied powers during World War II. The evidence of the epidemiological value of vectorproofing houses as a control measure against malaria was set aside and largely forgotten in favor of these two more attractive options. A half-century of experience has demonstrated how these two alternatives are incapable of the sort of global eradication that was envisaged at mid-century and have left researchers narrowing their hopes on vaccines as their remaining option for eradication. But broadly effective vaccines have proved elusive; moreover, the evolutionary versatility of plasmodia casts doubt on the long-term success of vaccination - the generation of effective vaccines may be a less formidable challenge than maintaining the efficacy of vaccines after they are put into use. The present therefore seems an opportune time to investigate the possibility of using the evolutionary versatility of plasmodia to our advantage, to generate milder variants.

Like Chagas, by quantifying the frequencies of cholera in areas of London one-and-one-half centuries ago, John Snow demonstrated that the frequencies of cholera were associated with contamination of water supplies (Snow 1855). By integrating evolutionary insights with this kind of epidemiological insight we can add a second dimension to studies of cholera, namely that the harmfulness of pathogens (and hence the harmfulness per infection) is also associated with contamination of water supplies.

Evolutionary considerations strengthen arguments for improving housing and water quality in two ways. First, evolutionary considerations reveal weak spots in programs based on insecticides and antibiotics: the target organisms evolve resistance. Second, evolutionary considerations suggest a previously unrecognized evolutionary benefit of such improvements: the target pathogens should evolve reduced virulence. To evaluate the validity of such evolutionary benefits the suggested interventions (making water supplies pure and houses vector-proof) need to be enacted and studied prospectively in human populations. The molecular and genetic tools are already available or could be readily developed for target pathogens in each category. Molecular determinants of virulence are needed to determine whether evolutionary changes in virulence occur. Molecular phylogenies are needed to categorize pathogens according to their epidemiological history and to structure statistical tests.

Our current state of knowledge already seems sufficient to justify such investments in these interventions ethically and economically. The experimental tracking of epidemiological changes in the frequencies of virulent and mild genotypes should provide conclusive answers to these evolutionary questions while simultaneously providing the epidemiological benefits envisioned by Snow and Chagas. 


\section{REFERENCES}

Ackerman EA 1956. TVA in its larger setting, p. 244256. In RC Martin, TVA. The First Twenty Years. A Staff Report, University of Alabama Press \& University of Tennessee Press.

Barrett VJ, Leiby DA, Odom JL, Otani MM, Rowe JD, Roote JT, Cox KF, Brown KR, Hoiles JA, Saez Alquezar A, Turrens JF 1997. Negligible prevalence of antibodies against Trypanosoma cruzi among blood donors in the southeastern United States. Am J Clin Pathol 108: 499-503.

Burkholder JE, Allison TC, Kelly VP 1980. Trypanosoma cruzi (Chagas) (Protozoa: Kinetoplastida) in invertebrate, reservoir, and human hosts of the lower Rio Grande Valley of Texas. J Parasitol 66: 305-311.

CDC - Centers for Disease Control 1996. Dengue fever at the U.S.-Mexico border, 1995-1996. Morb Mort Weekly Report 45: 841-844.

Clark CG, Pung OJ 1994. Host specificity of ribosomal DNA variation in sylvatic Trypanosoma cruzi from North America. Mol Biochem Parasitol 66: 175-179.

Dawson M, Johnson PT, Feldman L, Glover R, Koehler J, Blake P, Toomey KE 1997. Probable locally acquired mosquito-transmitted Plasmodium vivax infection - Georgia, 1996 (Reprinted from MMWR, vol 46, p. 264-267, 1997). JAMA 277: 1191-1193.

Derryberry OM 1956. Health, p. 193-205. In RC Martin, TVA. The First Twenty Years. A Staff Report, University of Alabama Press \& University of Tennessee Press.

Elhassan IM, Hviid L, Jakobsen PH, Giha H, Satti GMH, Arnot DE, Jensen JB, Theander TG 1995. High proportion of subclinical Plasmodium falciparum infections in an area of seasonal and unstable malaria in Sudan. Am J Trop Med Hyg 53: 78-83.

Ewald PW 1983. Host-parasite relations, vectors, and the evolution of disease severity. Ann Rev Ecol Syst 14: 465-85.

Ewald PW 1991. Waterborne transmission and the evolution of virulence among gastrointestinal bacteria. Epidemiol Infect 106: 83-119.

Ewald PW 1994. Evolution of Infectious Disease, Oxford University Press, New York.

Gonzalez JM, Olano V, Vergara J, Arevalo Herrera M, Carrasquilla G, Herrera S, Lopez JA 1997. Unstable, low-level transmission of malaria on the Colombian Pacific Coast. Ann Trop Med Parasitol 91: 349-358.

Harrison G 1978. Mosquitoes, Malaria \& Man: A History of the Hostilities Since 1880, EP Dutton, New York.

Harvey PH, Pagel MD 1991. The Comparative Method in Evolutionary Biology, Oxford University Press, Oxford.

Karaolis DKR, Lan R, Reeves PR 1995. The sixth and seventh cholera pandemics are due to independent clones separately derived from environmental, nontoxigenic, non-O1 Vibrio cholerae. J Bacteriol 177: 3191-3198.

Karsten V, Davis C, Kuhn R 1992. Trypanosoma cruzi in wild raccoons and opossums in North-Carolina. $J$ Parasitol 78: 547-549.

Kirchhoff LV 1993. Current concepts - American trypa- nosomiasis (Chagas' disease) - a tropical disease now in the United States. New Engl J Med 329: 639-644.

Kun JFJ, SchmidtOtt RJ, Lehman LG, Lell B, Luckner D, Greve B, Matousek P, Kremsner PG 1998. Merozoite surface antigen 1 and 2 genotypes and resetting of Plasmodium falciparum in severe and mild malaria in Lambarene, Gabon. Trans $R$ Soc Trop Med Hyg 92: 110-114.

Lauria Pires L, Teixeira ARL 1997. Protective effect of exposure to non-virulent Trypanosoma cruzi clones on the course of subsequent infections with highly virulent clones in mice. J Comp Pathol 117: 119-126.

Lenski RI, May RM 1994. The evolution of virulence in parasites and pathogens: reconciliation between two competing hypotheses. J Theor Biol 169: 253-265.

Morens DM, Marchette NJ, Chu MC, Halstead SB 1991. Growth of dengue type-2 virus isolates in human peripheral blood leukocytes correlates with severe and mild dengue disease. Am J Trop Med Hyg 45: 644-51.

Pung OJ, Banks CW, Jones DN, Krissinger MW 1995. Trypanosoma cruzi in wild raccoons, opossums, and triatomine bugs in southeast Georgia, U.S.A. $J$ Parasitol 81: 324-326.

Salles CA, Momen H 1991. Identification of Vibrio cholerae by enzyme electrophoresis. Trans $R$ Soc Trop Med Hyg 85: 544-547.

Snow J 1855 (1996 reprint). On the Mode of Communication of Cholera, 2nd ed., London, Churchill.

Snow RW, Marsh K 1995. Will reducing Plasmodium falciparum transmission alter malaria mortality among African children? Parasitol Today 11: 188190.

Tauxe RV, Mintz ED, Quick RE 1995. Epidemic cholera in the new world: translating field epidemiology in now prevention strategies. Emerg Infect Dis 1: 141-146.

Vaughn DW, Green S, Kalayanarooj S, Innis BL, Nimmannitya S, Suntayakorn S, Rothman AL, Ennis FA, Nisalak A 1997. Dengue in the early febrile phase: viremia and antibody responses. J Infect Dis 176: 322-330.

Watson RB 1949. Location and mosquito-proofing of dwellings, p. 1184-1202. In MF Boyd, Malariology. A Comprehensive Survey of All Aspects of This Group of Diseases from a Global Standpoint, Saunders, Philadelphia.

Weber JT, Levine WC, Hopkins DP, Tauxe RV 1994. Cholera in the United States, 1965-1991. Risks at home and abroad. Arch Intern Med 154: 551-556.

Weissman JB, Murton KI, Lewis JN, Friedemann CHT, Gangarosa EJ 1974. Impact in the U.S. of the Shiga dysentery pandemic of Central America and Mexico: A review of surveillance data through 1972. J Infect Dis 129: 218-23.

Woody NC, Woody HB 1961a. American trypanosomiasis I. Clinical and epidemiological background of Chagas' disease in the U.S. J Pediat 58: 568-580.

Woody NC, DeDranous N, Woody HB 1961b. American trypanosomiasis II. Current serologic studies in Chagas' disease. J Pediat 58: 738-745.

Wyler DJ 1993. Malaria: overview and update. Clin Infect Dis 16: 449-458. 